\title{
ANTERIOR CRUCIATE LIGAMENT RECONSTRUCTION USING THE DOUBLE-BUNDLE TECHNIQUE - EVALUATION IN THE BIOMECHANICS LABORATORY
}

Caio Oliveira D'Elia ${ }^{1}$, Alexandre Carneiro Bitar ${ }^{1}$, Wagner Castropil ${ }^{2}$, Antônio Guilherme Padovani Garofo ${ }^{3}$, Anita Lopes Cantuária ${ }^{4}$, Maria Isabel Veras Orselli ${ }^{5}$, Isabela Ugo Luques ${ }^{6}$, Marcos Duarte ${ }^{7}$

\section{ABSTRACT}

Objective: The objective of this study was to describe the methodology of knee rotation analysis using biomechanics laboratory instruments and to present the preliminary results from a comparative study on patients who underwent anterior cruciate ligament (ACL) reconstruction using the double-bundle technique. Methods: The protocol currently used in our laboratory was described. Threedimensional kinematic analysis was performed and knee rotation amplitude was measured on eight normal patients (control group) and 12 patients who were operated using the double-bundle technique, by means of three tasks in the biomechanics laboratory. Results: No significant differences between operated and non-operated sides were shown in relation to the mean amplitudes of gait, gait with change in direction or gait with change in direction when going down stairs $(p>0.13)$. Conclusion: The preliminary results did not show any difference in the double-bundle ACL reconstruction technique in relation to the contralateral side and the control group.

Keywords - Rotation; Anterior Cruciate Ligament; Reconstruction; Biomechanics

\section{INTRODUCTION}

The evaluation and measurement of the rotational movement that occurs in the knee joint, when compared to the measurement of the flexion and extension movement, is a complex measurement that is difficult to determine with accuracy in clinical practice. Different methods have been created and used to this end ${ }^{(1-4)}$.

Anterior cruciate ligament (ACL) reconstruction is a relatively common procedure ${ }^{(5)}$, and in spite of the evolution in the surgical technique, fixation materials, graft sources, and rehabilitation protocols, there is still a percentage of these patients (10-30\%) that do not evolve satisfactorily ${ }^{(6-9)}$.

One of the maneuvers used in the clinical evaluation of patients to assess rotational control of the knee is the pivot-shift test. This maneuver is extremely subjective and examiner-dependent. Reports of the presence of residual pivot-shift after ACL reconstruction by the single-bundle technique are not uncommon ${ }^{(6-8)}$.

\footnotetext{
1 - Master's Degree in Sciences, Orthopedic Doctor at the Instituto Vita, SP.

2 - Master's Degree and PhD in Sciences, Orthopedist at the Instituto Vita, SP.

3 - Orthopedic Doctor at the Instituto Vita, SP.

4 - Physiotherapist at the Instituto Vita, SP.

5 - Master's Degree in Sciences; Instituto de Física da USP, SP.

6 - Master's Degree in Human Anatomy from USP, Researcher at the Instituto Vita, SP

7 - Doctorate Professor at the School of Physical Education and Sport, USP, SP
}

Work carried out at the Instituto Vita, São Paulo.

Correspondence: Instituto Vita, Rua Mato Grosso, 306, $1^{\circ}$ andar, Higienópolis - 01239-040 - São Paulo, SP, A/C.: Isabela Ugo Luques. E-mail: isabela@vita.org.br 
The ACL is composed of two well-defined bundles (or bands): the anteromedial (AM) and posterolateral (PL) bundles. Biomechanical studies on cadavers have already shown that the surgical technique that reconstructs both ACL bundles is able to offer better control over anterior translation of the tibia, as well as better control of knee rotation resulting, in theory, from posterolateral bundle reconstruction ${ }^{(9-13)}$.

Clinical trials have already shown that the double-bundle technique offers better control of anterior translation of the tibia under the femur, measurements that are obtained through the physical examination, using the clinical tests of anterior drawer, Lachman and KT1000 or KT $2000^{(14-16)}$ manual arthrometry.

The correct evaluation and comparison of the double-bundle ACL reconstruction technique with the single-bundle technique, which is still considered standard, calls for the development of a method that analyzes knee rotation more accurately and objectively.

The aim of this study is to describe a knee rotation analysis methodology using instruments present in a biomechanics laboratory, and to present the preliminary results of a study in which patients were submitted to ACL reconstruction with the doublebundle technique.

\section{MATERIALS AND METHODS}

\section{1) Analysis of rotation}

The tasks proposed in this study represent a minimal risk to the subjects' health. There is no conflict of interests in the performance of this study, which only began after obtaining approval from the Science and Ethics Committee of Vita Care. All the subjects signed a Informed Consent Form.

\section{1) Data Collection}

Six infrared video cameras (VICON) with acquisition frequency of $250 \mathrm{~Hz}$ were used for three-dimensional kinematic analysis, while an OR6 model (AMTI) force platform was used to acquire the kinetic data, particularly the vertical ground reaction force. The lower limb kinematic analysis protocol was standardized within previously proposed and established models ${ }^{(17)}$.

Retro-reflective markers were placed on anatomical prominences at the following locations of the body: right and left anterior superior iliac spine, greater trochanter, lateral and medial femoral epicondyle, apex of head of fibula, anterior tibial tuberosity, and distal apex of the lateral and medial malleoli ${ }^{(18)}$.

To minimize measurement errors of the kinematic data, we used the calibrated anatomical system technique (CAST) proposed by Cappozzo et $\mathrm{al}^{(17)}$. In the use of CAST, the first stage of collection consists of a static calibration in which the subject remains stationary in the anatomical position, aligned in the direction in which they will execute the task. Besides the anatomical markers, the subjects used another two sets of markers, one on the thigh and the other on the leg (Figure 1). This procedure was adopted to define the position of the markers in relation to the base of the sets of markers (clusters). Once the positions of the markers were defined, these were removed and the subjects carried out the proposed tasks using just the clusters.

Assuming that the thigh and leg segments are rigid bodies, and that the clusters do not move in relation to the markers of the respective segments, the position vector of the markers in relation to the respective cluster does not move as the segment moves. Therefore, with the static calibration, all the position vectors of the thigh and leg markers are determined and described from the local coordinate system, enabling the markers to be removed while the tasks are carried out ${ }^{(17)}$.

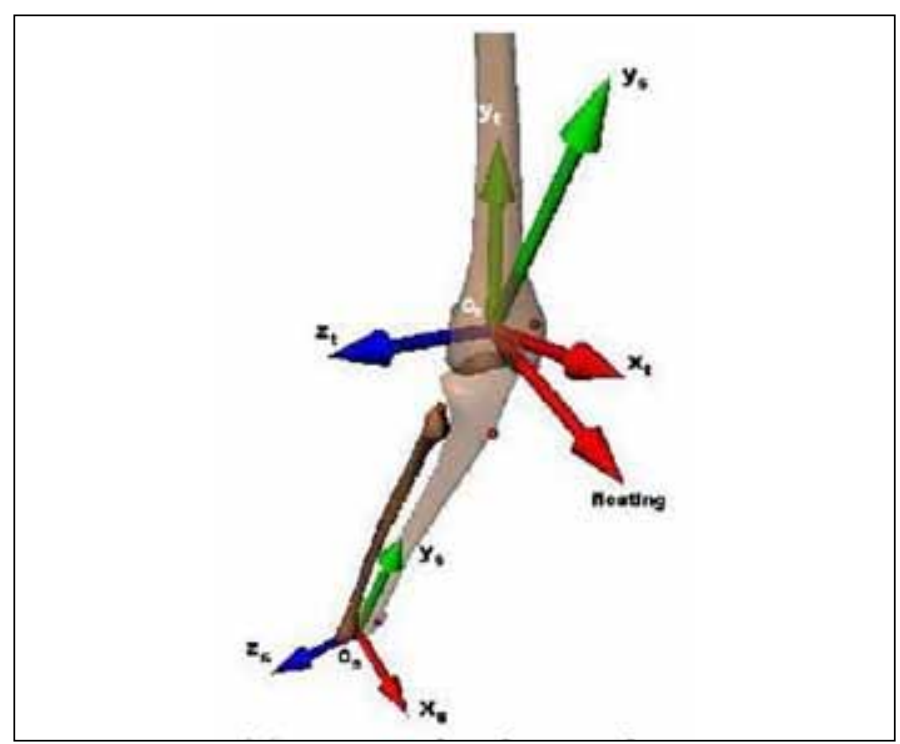

Figure 1 - Positioning of the clusters, of the anatomical markers (left) and the convention adopted for measuring the joint angles (right). 
During the performance of the tasks, only the clusters are used in the leg and thigh segment. Based on the clusters, the positions of the withdrawn markers are reconstructed virtually by means of computational routines written in the Matlab environment (version 6.5, Mathworks Inc.) using the position vectors obtained in the static calibration.

\section{2) Data analysis}

The position of the axes and joint planes (anatomical basis) were determined as described by Cappozzo et $\mathrm{al}^{(18)}$. It is necessary to determine the centers of the hip, knee and ankle joints in order to define the joint axes. The coordinates of the hip joint center $\left(x_{c}, y_{c}\right.$ and $z_{C}$ ) are determined by the hybrid method reported by Andriacchi et al ${ }^{(19)}$. The knee joint center is considered the midpoint between the femoral epicondyles, and the ankle joint center as the midpoint between the lateral and medial malleoli (Figure 2).



Figure 2 - Knee joint coordinates system.

All the angles were calculated in relation to the static position, in other words, the variables of the dynamic attempt were referenced in relation to the anatomical calibration. Figure 1 shows the position of the retro-reflective markers positioned on the anatomical prominences of the subjects, as well as the two clusters used in each segment, and the convention adopted in this study to measure the joint angles.

\section{3) Tasks}

The objective of the tasks was to imitate functional movements of the knee and to evaluate its rotation in tasks of increasing difficulty.

Rev Bras Ortop. 2011;46(2):148-54
The tasks used were:

\section{Walking without change in direction}

In this task, the individual is asked to walk along at a comfortable speed, in a straight line, so that one of their feet touches the center of the force platform inserted in the laboratory floor. The participant repeats the task until they touch the platform correctly at least five times with each foot. The data referring to the stance phase of gait are then analyzed.

\section{Walking with change in direction}

To perform this task, the patient is asked to walk along in a straight line until one of their feet touches the force platform. At this moment the participant should change their direction of movement at an angle of $90^{\circ}$ to their original trajectory, rotating to the side of the foot that will touch the platform. Thus, when the patient touches the platform with their right foot, they will shift $90^{\circ}$ to the right, and when the left foot touches the platform, $90^{\circ}$ to the left.

As in the first task, this task is repeated until it is performed correctly at least five times for each side. The joint angles are analyzed during the single stance phase (from initial contact of one of the heels to contact of the contralateral heel) of this task.

\section{Staircase descent with change in direction}

To evaluate the staircase descent, we used a staircase specially designed for this experiment. It has four steps, and dimensions that are similar to those used by Georgoulis et $\mathrm{al}^{(20)}$ and Andriacchi et $\mathrm{al}^{(19)}$ in their studies (Figure 3).

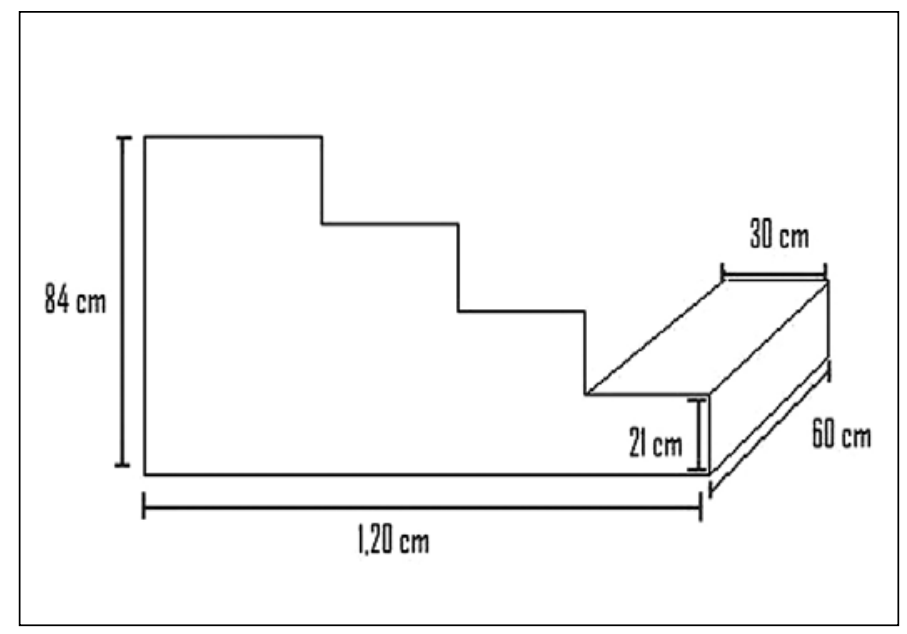

Figure 3 - Diagram of staircase used to evaluate knee biomechanics during staircase descent with change in direction. 
The patients are asked to descend the four steps and, on touching the ground, where the force platform is located, to change their direction of movement, so that the new trajectory forms an angle of $90^{\circ}$ with the old one (Figure 4).

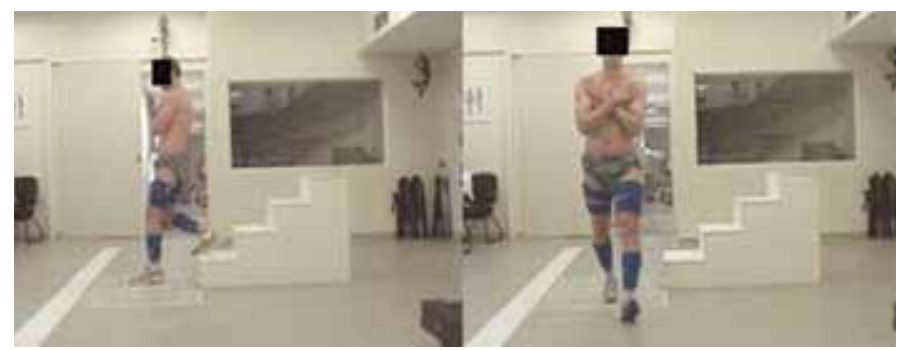

Figure 4 - Touchdown with change in direction task.

In this task the joint angles are also analyzed during the single stance phase (from initial contact of one of the heels to contact of the contralateral heel). The rotation of both knees is evaluated in each patient (operated and not operated) in the three tasks.

\section{2) Patients studied}

The data presented below refer to the knee rotation amplitude measurements (measurements in degrees) in the walking $(\mathrm{G})$, walking with change in direction (gait WC) and walking with change in direction while descending stairs (S) tasks. We assessed eight normal patients (control group) and 12 patients operated using the double-bundle technique.

The control group was comprised of eight $(\mathrm{N}=8)$ individuals without previous surgery or any complaint regarding the knee joints.

The study group was comprised of $12(\mathrm{~N}=12)$ patients operated using the double-bundle technique, and that had been medically discharged to carry out their activities, including sports, after clinical evaluation and imaging exams (magnetic resonance).

In relation to the control group, we compared the knee rotation amplitude (in degrees) between the right and left knees.

In the study group, we compared the rotation amplitude (in degrees) between the operated knee and the normal contralateral side, and between the operated knee and the values obtained in the control group.

The patients of the control and study groups were similar in terms of body mass index (BMI) and age (Table 1).

The surgical technique consisted of anatomic ACL reconstruction, using autologous graft from the flexor tendons fixed in two tibial tunnels and two independent femoral tunnels ${ }^{(21)}$ (Figure 5).

Table 1 - Data relating to the BMI and age of the patients evaluated.

\begin{tabular}{l|l|c|c|c|c|c|c}
\hline BMI & Control & 8 & 23.8 & 2.2 & 24.1 & 19.1 & 26.7 \\
\hline $\mathrm{p}=0.193$ & $\begin{array}{l}\text { Double } \\
\text { bundle }\end{array}$ & 12 & 25.6 & 3.8 & 25.3 & 20.9 & 31.0 \\
\hline AGE & Total & 20 & 24.9 & 3.3 & 24.1 & 19.1 & 31.0 \\
\hline $\mathrm{p}=0.572$ & Control & 8 & 26.3 & 8.2 & 23.0 & 21.0 & 46.0 \\
\hline & $\begin{array}{l}\text { Double } \\
\text { bundle }\end{array}$ & 12 & 24.5 & 5.5 & 24.0 & 16.0 & 33.0 \\
\hline
\end{tabular}

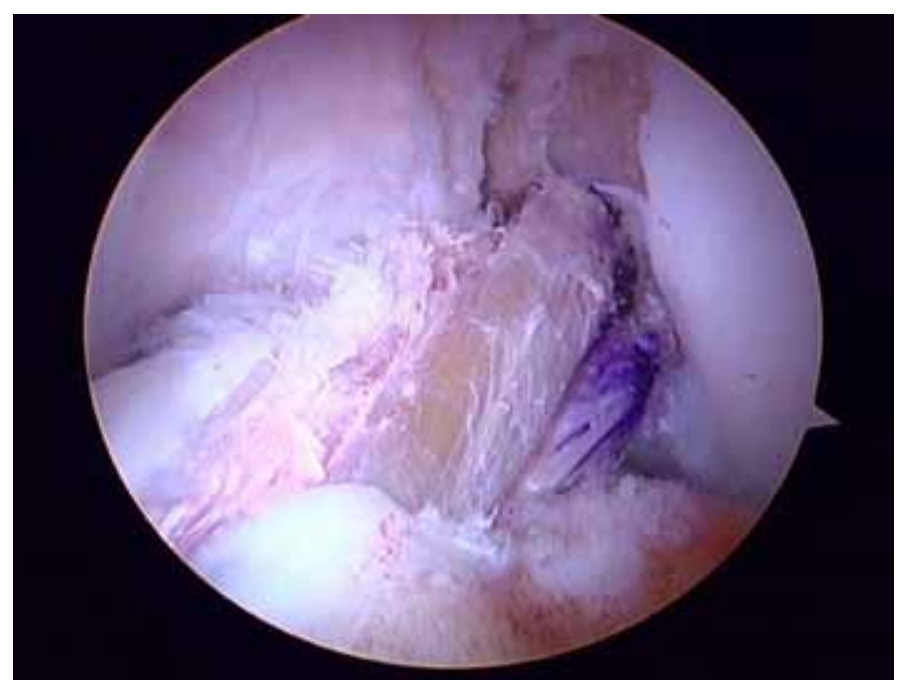

Figure 5 - Arthroscopic view of $A C L$ reconstruction using the double-bundle technique.

\section{RESULTS}

No differences were observed in the rotation amplitudes between the left and right knees in the individuals of the group control, in any of the three tasks performed (Table 2).

During the analysis of the study group, we detected two flaws in the data collection (one in the staircase task - patient no. 8, and another in the change of direction - patient no. 11). For this reason, differences are noted in the number of patients evaluated in Tables 2 to 4 .

Three patients in the study group (no. 3, no. 4 and no. 9) had undergone previous ACL reconstruction surgery using single-bundle technique on the contralateral knee, hence there is a noticeable difference 
Table 2 - Comparison between right and left knees within the control group.

\begin{tabular}{l|l|l|l}
\hline & & Mean & SD \\
\hline Stairs & Right & 30.0 & 4.7 \\
\hline $\mathrm{p}=0.593$ & Left & 31.6 & 8.0 \\
\hline Gait WC & Right & 26.4 & 5.3 \\
\hline $\mathrm{p}=0.834$ & Left & 26.9 & 6.4 \\
\hline Gait & Right & 12.4 & 4.1 \\
\hline $\mathrm{p}=0.949$ & Left & 12.5 & 4.7 \\
\hline$p$ value for the paired
\end{tabular}

$\mathrm{p}$ value for the paired $t$-test $(\mathrm{n}=8$ individuals)

in the number of patients evaluated in Table 2 (in which we compared only the patients operated on one knee with the uninjured contralateral knee) compared with Tables 3 and 4, in which the comparison was made between the knee operated on using the double-bundle technique and the data obtained from the control group.

No difference was found in mean knee rotation amplitude between the knees operated with the double-bundle technique, and the uninjured contralateral knee (Tables 3, 4 and 5).

Table 3 - Comparison between knees operated with doublebundle technique and the non-operated contralateral knees (i.e. disregarding patients no.3, no.4 and no.9).

\begin{tabular}{l|l|c|c|c|c|c}
\hline & & Mean & SD & Median & Minimum & Maximum \\
\hline $\begin{array}{l}\text { Stairs }(\mathrm{N}=8 \\
\text { patients })\end{array}$ & Contralateral & 28.4 & 7.6 & 30.0 & 16.7 & 38.2 \\
\hline $\mathrm{p}=0.199$ & Operated & 26.0 & 5.2 & 26.0 & 17.3 & 34.4 \\
\hline $\begin{array}{l}\text { Gait WC } \\
(\mathrm{N}=8 \text { patients) }\end{array}$ & Contralateral & 27.9 & 5.6 & 27.0 & 20.0 & 39.3 \\
\hline $\mathrm{p}=0.131$ & Operated & 24.5 & 5.8 & 24.1 & 17.7 & 36.8 \\
\hline $\begin{array}{l}\text { Gait } \\
(\mathrm{N}=9 \text { patients) }\end{array}$ & Contralateral & 12.4 & 3.2 & 12.2 & 8.0 & 19.3 \\
\hline $\mathrm{p}=0.264$ & Operated & 11.8 & 2.9 & 10.7 & 8.3 & 16.8 \\
\hline
\end{tabular}

$p$ value for the paired t-test.

\section{DISCUSSION}

ACL lesions have an extremely negative impact on the knee joint. It is known that the individual who suffers an ACL rupture has a higher incidence of osteoarthritis $(\mathrm{OA})^{(22)}$. Among the various factors invol- ved in the genesis of OA after ACL rupture, one of the most extensively studied is the mechanical factor, caused by loss of joint stability ${ }^{(23,24)}$. Even with modern reconstruction techniques, it has not yet proven possible to demonstrate a lower incidence of OA in operated individuals ${ }^{(25,26)}$.

The presence of residual pivot-shift after ACL reconstruction surgery appears to be more closely correlated to the presence of OA than to the actual anterior instability. The clinical evaluation performed with the pivot-shift test seeks to evaluate the degree of rotational instability caused by the ACL lesion. As we know, this is an examiner-dependent and relatively subjective measurement.

The biomechanics laboratory has proven to be an effective and reliable tool in the evaluation of knee joint movements. The method used in this study is validated and confers greater objectivity in the evaluation of knee joint movements ${ }^{(17,18)}$.

The technique considered standard in ACL reconstruction is the single-bundle technique. This technique is very efficient in controlling tibial translation under the femur, but is not as efficient in controlling tibial rotation under the femur. Kinematic studies have demonstrated that ACL reconstruction with single-bundle technique is unable to reestablish the normal kinematics of the joint ${ }^{(9,13)}$.

One of the methods for evaluating rotational control in the knee joint is the measurement of total rotation amplitude. The normal contralateral knee has been frequently used as a parameter of normality to evaluate the result of ACL reconstruction.

In this study, we verified that ACL reconstruction with the double-bundle technique was efficient in relation to knee rotation control. With regard to the results obtained in the sample, considering patients operated using the double-bundle technique, these do not indicate any significant differences between operated and non-operated sides in relation to mean amplitudes in the walking, walking with change in direction, and staircase descent with change in direction $(p>0.13)$ tests. Neither did these mean amplitudes showed no differences when comparing the control and study groups $(\mathrm{p}>0.05)$.

The methodology used here allows the evaluation of patients with multi-ligament lesions, angular and/ or torsional deformities of the lower limbs, and any 
Table 4 - Comparison between the measurements of the knees operated using double-bundle technique and the summary measurements of the knees from the control group (i.e., the mean amplitude of the right and left knee is considered for each patient of the control group).

\begin{tabular}{|c|c|c|c|c|c|c|c|}
\hline & Group & $\mathbf{N}$ & Mean & SD & Median & Minimum & Maximum \\
\hline Stairs & Control & 8 & 30.8 & 5.1 & 29.9 & 22.8 & 38.5 \\
\hline \multirow[t]{2}{*}{$p=0.088$} & Double-bundle & 11 & 26.4 & 5.2 & 27.1 & 17.3 & 34.4 \\
\hline & Total & 19 & 28.2 & 5.5 & 28.6 & 17.3 & 38.5 \\
\hline Gait WC & Control & 8 & 26.7 & 5.0 & 26.4 & 19.0 & 33.3 \\
\hline \multirow[t]{2}{*}{$p=0.756$} & Double-bundle & 11 & 25.8 & 6.8 & 24.2 & 17.7 & 39.9 \\
\hline & Total & 19 & 26.1 & 6.0 & 26.1 & 17.7 & 39.9 \\
\hline Gait & Control & 8 & 12.5 & 3.1 & 12.0 & 8.5 & 17.6 \\
\hline \multirow[t]{2}{*}{$p=0.423$} & Double-bundle & 12 & 11.4 & 2.7 & 10.5 & 8.3 & 16.8 \\
\hline & Total & 20 & 11.8 & 2.8 & 11.1 & 8.3 & 17.6 \\
\hline
\end{tabular}

$\mathrm{p}$ value for the student's t-test, with $\mathrm{p} 1$ for the test corrected due to inequality of variances.

Table 5 - Descriptive table of amplitudes for the knees operated using the double-bundle technique according to dominant leg

\begin{tabular}{|c|c|c|c|c|c|c|c|}
\hline & Dominance & $\mathbf{N}$ & Mean & SD & Median & Minimum & Maximum \\
\hline \multirow[t]{3}{*}{ Stairs } & No & 3 & 25.9 & 6.9 & 22.3 & 21.7 & 33.9 \\
\hline & Yes & 8 & 26.6 & 5.0 & 27.2 & 17.3 & 34.4 \\
\hline & Total & 11 & 26.4 & 5.2 & 27.1 & 17.3 & 34.4 \\
\hline \multirow[t]{3}{*}{ Gait WC } & No & 3 & 26.6 & 11.7 & 22.3 & 17.7 & 39.9 \\
\hline & Yes & 8 & 25.4 & 5.1 & 24.8 & 19.8 & 36.8 \\
\hline & Total & 11 & 25.8 & 6.8 & 24.2 & 17.7 & 39.9 \\
\hline \multirow[t]{3}{*}{ Gait } & No & 3 & 10.7 & 2.6 & 10.2 & 8.4 & 13.6 \\
\hline & Yes & 9 & 11.6 & 2.8 & 10.7 & 8.3 & 16.8 \\
\hline & Total & 12 & 11.4 & 2.7 & 10.5 & 8.3 & 16.8 \\
\hline
\end{tabular}

other clinical situation that might appear in association with knee rotation alterations. This type of evaluation may become another instrument to assist in decision making in the treatment of these patients in the future.

Although we used a validated methodology, we applied it to a group of patients that had never been evaluated by this method (control individuals and patients submitted to reconstruction with double-bundle technique). Our results should, therefore, be viewed as merely indicative, due to our restricted number of cases. Since 2006, our group has already carried out
87 double-bundle ACL reconstructions; these patients will be involved in our evaluations, thus increasing our case studied.

\section{CONCLUSIONS}

We believe that the biomechanics laboratory is a useful tool for objectively evaluating knee rotation. The preliminary results do not show any difference of the double-bundle ACL reconstruction technique in relation to the contralateral side or the control group. 


\section{REFERENCES}

1. Ishibashi Y, Tsuda E, Tazawa K, Sato H, Toh S. Intraoperative evaluation of the anatomical double-bundle anterior cruciate ligament reconstruction with the OrthoPilot navigation system. Orthopedics. 2005;28(10 Suppl):1277-82.

2. Ishibashi $Y$, Tsuda E, Yamamoto $Y$, Tsukada H, Toh S. Navigation evaluation of the pivot-shift phenomenon during double-bundle anterior cruciate ligament reconstruction: is the posterolateral bundle more important? Arthroscopy. 2009;25(5):488-95.

3. Ristanis S, Giakas G, Papageorgiou CD, Moraiti T, Stergiou N, Georgoulis $A D$. The effects of anterior cruciate ligament reconstruction on tibial rotation during pivoting after descending stairs. Knee Surg Sports Traumatol Arthrosc. 2003;11(6):360-5

4. Tashman S, Collon D, Anderson K, Kolowich P, Anderst W. Abnormal rotational knee motion during running after anterior cruciate ligament reconstruction. Am J Sports Med. 2004;32(4):975-83.

5. Brown $\mathrm{CH}$ Jr, Carson EW. Revision anterior cruciate ligament surgery. Clin Sports Med. 1999;18(1):109-71.

6. Lubowitz JH, Poehling GG. Techniques in double-bundle anterior cruciate ligament reconstruction: as simple as $A B C$, or putting the cart before the horse? Arthroscopy. 2008;24(10):1089-91.

7. Muneta T, Koga H, Morito T, Yagishita K, Sekiya I. A retrospective study of the midterm outcome of two-bundle anterior cruciate ligament reconstruction using quadrupled semitendinosus tendon in comparison with one-bundle reconstruction. Arthroscopy. 2006;22(3):252-8.

8. Yasuda K, Kondo E, Ichiyama H, Tanabe $\mathrm{Y}$, Tohyama H. Clinical evaluation of anatomic double-bundle anterior cruciate ligament reconstruction procedure using hamstring tendon grafts: comparisons among 3 different procedures. Arthroscopy. 2006;22(3):240-51.

9. Gabriel MT, Wong EK, Woo SL, Yagi M, Debski RE. Distribution of in situ forces in the anterior cruciate ligament in response to rotatory loads. J Orthop Res. 2004;22(1):85-9.

10. Mae T, Shino K, Miyama T, Shinjo H, Ochi T, Yoshikawa H, et al. Single-versus two-femoral socket anterior cruciate ligament reconstruction technique: Biomechanical analysis using a robotic simulator. Arthroscopy. 2001;17(7):708-16.

11. Mochizuki T, Muneta T, Nagase T, Shirasawa S, Akita KI, Sekiya I. Cadaveric knee observation study for describing anatomic femoral tunnel placement for two-bundle anterior cruciate ligament reconstruction. Arthroscopy. 2006;22(4):356-61.

12. Yagi M, Wong EK, Kanamori A, Debski RE, Fu FH, Woo SL. Biomechanical analysis of an anatomic anterior cruciate ligament reconstruction. Am J Sports Med. 2002;30(5):660-6.

13. Zantop T, Herbort M, Raschke MJ, Fu FH, Petersen W. The role of the anteromedial and posterolateral bundles of the anterior cruciate ligament in anterior tibial translation and internal rotation. Am J Sports Med. 2007;35(2):223-7.
14. Adachi N, Ochi M, Uchio Y, Iwasa J, Kuriwaka M, Ito Y. Reconstruction of the anterior cruciate ligament. Single- versus double-bundle multistranded hamstring tendons. J Bone Joint Surg Br. 2004;86(4):515-20.

15. Hamada M, Shino K, Horibe S, Mitsuoka T, Miyama T, Shiozaki Y, et al. Singleversus bi-socket anterior cruciate ligament reconstruction using autogenous multiple-stranded hamstring tendons with endoButton femoral fixation: A prospective study. Arthroscopy. 2001;17(8):801-7.

16. Siebold R, Dehler C, Ellert T. Prospective randomized comparison of doublebundle versus single-bundle anterior cruciate ligament reconstruction. Arthroscopy. 2008;24(2):137-45.

17. Cappozzo A, Catani F, Leardini A, Benedetti MG, Croce UD. Position and orientation in space of bones during movement: experimental artefacts. Clin Biomech (Bristol, Avon). 1996;11(2):90-100.

18. Cappozzo A, Catani F, Croce UD, Leardini A. Position and orientation in space of bones during movement: anatomical frame definition and determination. Clin Biomech (Bristol, Avon). 1995;10(4):171-8.

19. Andriacchi TP, Andersson GB, Fermier RW, Stern D, Galante JO. A study of lower-limb mechanics during stair-climbing. J Bone Joint Surg Am. 1980;62(5):749-57.

20. Georgoulis AD, Papadonikolakis A, Papageorgiou CD, Mitsou A, Stergiou $\mathrm{N}$. Three-dimensional tibiofemoral kinematics of the anterior cruciate ligament-deficient and reconstructed knee during walking. Am J Sports Med. 2003;31(1):75-9.

21. Jarvela T, Moisala AS, Sihvonen $R$, Jarvela $S$, Kannus $P$, Jarvinen M. Doublebundle anterior cruciate ligament reconstruction using hamstring autografts and bioabsorbable interference screw fixation: prospective, randomized, clinical study with 2-year results. Am J Sports Med. 2008;36(2):290-7.

22. Shefelbine SJ, Ma CB, Lee KY, Schrumpf MA, Patel P, Safran MR, et al. MRI analysis of in vivo meniscal and tibiofemoral kinematics in ACL-deficient and normal knees. J Orthop Res. 2006;24(6):1208-17.

23. Logan MC, Williams A, Lavelle J, Gedroyc W, Freeman M. Tibiofemoral kinematics following successful anterior cruciate ligament reconstruction using dynamic multiple resonance imaging. Am J Sports Med. 2004;32(4):984-92.

24. Tashman S, Collon D, Anderson K, Kolowich P, Anderst W. Abnormal rotational knee motion during running after anterior cruciate ligament reconstruction. Am J Sports Med. 2004;32(4):975-83.

25. Jomha NM, Borton DC, Clingeleffer AJ, Pinczewski LA. Long-term osteoarthritic changes in anterior cruciate ligament reconstructed knees. Clin Orthop Relat Res. 1999;(358):188-93.

26. Brandsson S, Karlsson J, Sward L, Kartus J, Eriksson BI, Kärrholm J. Kinematics and laxity of the knee joint after anterior cruciate ligament reconstruction: pre- and postoperative radiostereometric studies. Am J Sports Med. 2002;30(3):361-7. 